\title{
Comparison of superb microvascular imaging (SMI) quantified with ImageJ to quantified contrast-enhanced ultrasound (qCEUS) in liver metastases - a pilot study
}

\author{
Wolfgang Kratzer" ${ }^{1 \#}$, Melanie Güthle ${ }^{1 \#}$, Felix Dobler ${ }^{1}$, Thomas Seufferlein ${ }^{1}$, Tilmann Graeter ${ }^{2}$, \\ Julian Schmidberger ${ }^{1}$, Thomas FE Barth ${ }^{3}$, Jochen Klaus ${ }^{1}$ \\ ${ }^{1}$ Department of Internal Medicine I, University Hospital Ulm, Ulm, Germany; ${ }^{2}$ Department of Diagnostic and Interventional Radiology, University \\ Hospital Ulm, Ulm, Germany; ${ }^{3}$ Institute of Pathology, University Hospital Ulm, Ulm, Germany
}

Contributions: (I) Conception and design: W Kratzer, M Güthle, J Klaus; (II) Administrative support: W Kratzer, T Seufferlein, J Klaus; (III) Provision of study materials or patients: M Güthle, T Graeter, TFE Barth; (IV) Collection and assembly of data: F Dobler, J Schmidberger; (V) Data analysis and interpretation: F Dobler; (VI) Manuscript writing: All authors; (VII) Final approval of manuscript: All authors.

"These authors contributed equally to this work.

Correspondence to: Wolfgang Kratzer, MD. Department of Internal Medicine I, University Hospital Ulm, Albert-Einstein-Allee 23, 89081 Ulm, Germany. Email: wolfgang.kratzer@uniklinik-ulm.de.

Background: The aim of the study was to compare methods for the assessment of vascularisation of liver metastases (LM) between superb microvascular imaging (SMI), contrast-enhanced ultrasound, and microvascular density (MVD).

Methods: SMI results were quantified as the vascularisation quotient (VQ), based on a grey-scale analysis with ImageJ image software. Those results were compared to contrast-enhanced ultrasonography (CEUS) values, calculated with VueBox ${ }^{\circledR}$. MVD was measured with an anti-CD34 antibody.

Results: This study included 13 patients with LM. The VQ showed a strong correlation with the quantified parameters of contrast-enhanced ultrasound. The parameters of quantified contrast-enhanced ultrasound compared with quantified SMI showed the following statistical correlations: peak enhancement (PE), in arbitrary unit (a.u.) ( $\mathrm{r}=0.72104, \mathrm{P}=0.0054)$, $\mathrm{PE}$ in Decibel (dB) (r=0.65918, $\mathrm{P}=0.00141)$, Wash-inArea Under the Curve (WiAUC) in a.u. ( $\mathrm{r}=0.63604, \mathrm{P}=0.00194)$, Wash-in Perfusion-Index (WiPI) in a.u. ( $\mathrm{r}=0.73337, \mathrm{P}=0.0043)$, Wash-in Perfusion-Index (WiPI) in $\mathrm{dB}(\mathrm{r}=0.65642, \mathrm{P}=0.0194)$, Wash-in-Rate (WiR) in a.u. $(\mathrm{r}=0.7304, \mathrm{P}=0.0036)$ and Wash-in-Rate $(\mathrm{WiR})$ in $\mathrm{dB}(\mathrm{r}=0.82897, \mathrm{P}=0.0005)$.

Conclusions: Comparison of the two methods, SMI and contrast-enhanced ultrasound (CEUS), for quantitative assessment of vascularisation of LM showed good correlation. The contrast-independent Doppler technique SMI can qualitatively assess the vascularisation of LM.

Keywords: Liver; metastasis; Doppler ultrasound; computer-assisted image analyses; contrast-enhanced ultrasound

Submitted April 07, 2021. Accepted for publication Oct 12, 2021.

doi: 10.21037/qims-21-383

View this article at: https://dx.doi.org/10.21037/qims-21-383 


\section{Introduction}

It remains challenging to use B-scan ultrasound to differentiate focal liver lesions (FLLs), such as focal nodular hyperplasia, adenomas from hepatocellular and biliary carcinomas, and metastases (1). The early detection of liver metastases $(\mathrm{LM})$ is important for therapy. LMs are found in $25-50 \%$ of patients with known carcinoma (2). Contrastenhanced ultrasound (CEUS), computed tomography (CT), and magnetic resonance imaging (MRI) have similar detection rates for the diagnosis of LM (3). However, all of these methods have limitations (4). For example, CT requires contrast media that contains iodine, which can lead to thyroid and kidney problems $(5,6)$. The gadoliniumbased contrast agents used in MRI can lead to nephrogenic systemic fibrosis, and contrast agent can accumulate in the brain $(7,8)$. Furthermore, both CT and MRI contrast media can lead to significant allergic reactions (9). As with cross-sectional imaging, MRI, and CT, CEUS also requires intravenous access (4).

Nevertheless, CEUS data provides crucial information on LM vascularisation pattern. Metastases are often identified in CEUS based on the observation of hypervascularisation in the arterial phase and washout phenomena in the late phase (10). Despite the limitation, the assessment of the flow of the contrast medium in the arterial phase remains a decisive finding in the characterization of liver lesions; the malignancy of a liver lesion cannot be determined solely on the basis of the arterial phase (11).

A further advantage of contrast-enhanced ultrasound, however, remains the flooding of the contrast medium, which makes a decisive contribution to the characterization of liver lesions. In particular, the late washout, for example in highly differentiated hepatocellular carcinomas, should be mentioned here (12). CEUS is a powerful diagnostic tool for the assessment of liver lesions and is easier to use in renal failure, hyperthyroidism, pregnancy and in pediatric patients compared to CT scans due to its lower side effect profile $(13,14)$. Contrast-enhanced ultrasound can be performed by using a continuous microbubble infusion or by injection of a single bolus. Since the determination of the optimal infusion rate is difficult and an infusion rate that is too low or too high has a significant effect on the signal to noise ratio, the bolus administration currently presents the most commonly used form of contrast agent administration for non-destructive CEUS imaging (15).

Quantification of perfusion parameters is currently the subject of several studies. Moreover, CEUS could potentially miss heterogeneous vascularisation in metastases due to its two-dimensional determination of metastatic vascularisation $(16,17)$. On the other hand, Dujardin et al. demonstrated a very good reproducibility for three different ROIs in LM in patients with colorectal cancer in a recent CEUS quantification study (18). Furthermore, the tools currently available for CEUS quantification remain susceptible to artefacts $(10,15,18)$.

A potential alternative method is based on superb microvascular imaging (SMI), an image-processing technique developed by Toshiba/Canon (Minato, Tokyo, Japan) (19). SMI is an advanced ultrasound technology that filters the different frequency spectrum signals generated by tissue motion artifacts and displays microvascular flow through an adaptive algorithm that removes clutter dramatically (20).

SMI uses advanced clutter suppression to extract blood flow signals from small and large vessels and it presents this information at a high image repetition rate. SMI can be used in two modes, colour superb microvascular imaging (cSMI) and monochrome superb microvascular imaging (mSMI) (19). SMI has been used in several previous studies on the differentiation of FLLs. In a recent work in semiquantitative comparison between color Doppler flow imaging (CDFI) and SMI, the group of Yang et al. confirmed a superiority in the detection of microvascular blood flow signals in HCC's. When comparing SMI with color flow Doppler (CFD) and power Doppler (PD) imaging in the liver to differentiate between malignant and benign liver neoplasms, Dubinsky et al. demonstrated more central and peripheral vessels around liver lesions on SMI than on CFD and PD imaging (21). There were significantly more vessels in the periphery of malignant liver lesions than benign lesions. Those studies have described its usefulness, but the value of this method has been mainly studied semi-quantitatively $(21,22)$. With $\mathrm{mSMI}$, it is possible to quantify the differences in brightness, according to the vascularisation of the FLL, compared to the surrounding liver parenchyma. This analysis can be performed with ImageJ, an image analysis program used in the biological sciences and other fields (23). Quantitative SMI has several advantages over quantitative CEUS. No contrast agent is required for SMI. In addition, due to the progressive destruction of the phospholipid microbubbles over time, there is a time limit on CEUS imaging evaluated with SonoVue ${ }^{\circledR}$, whereas the informative value of SMI is not influenced by time. Another approach for assessing FLL vascularisation is to determine microvascular 


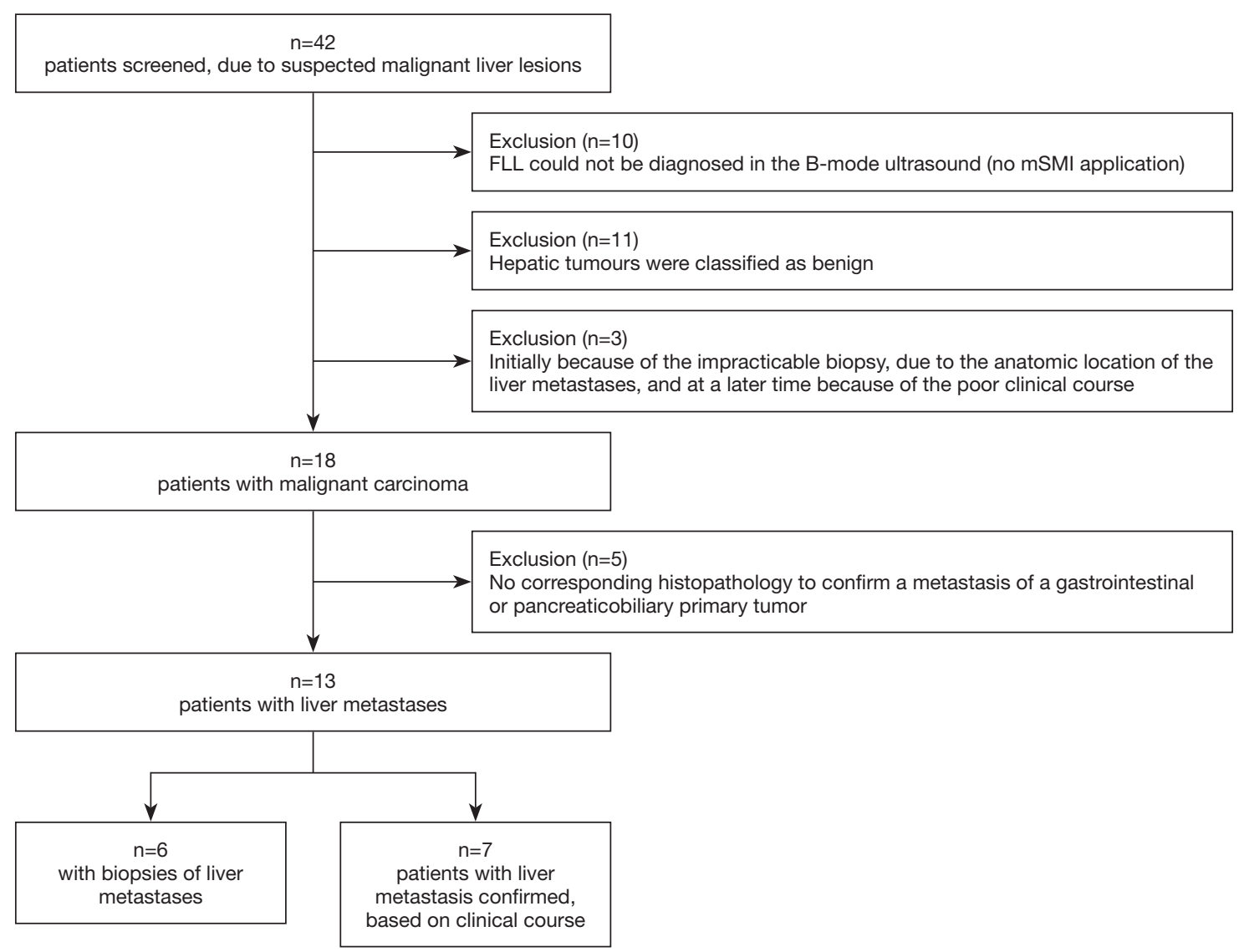

Figure 1 Flow chart shows the patient screening and selection process.

density (MVD) (24). Previous investigations have used immunohistochemical assays for this purpose. Antibodies that specifically recognize CD34 or CD31 on endothelial cells have been used to investigate vascularisation. Studies have shown good correlations between imaging-determined vascularity and immunohistologically determined vessel density $(25,26)$.

The main objective of the present pilot study was to compare methods for the assessment of vascularisation of metastases between SMI, quantified using the software program ImageJ and contrast-enhanced sonography (CEUS), with the ones quantified using the software tool VueBox ${ }^{\circledR}$. in LM confirmed by clinical course, or partially histologically. Furthermore, in cases of histologically confirmed LM and the presence of a tissue sample, we investigated whether quantifiable perfusion could be detected by immunohistochemistry using anti-CD34 antibodies.

\section{Methods}

\section{Patients}

The present study is a prospective pilot observational study. From March 2016 to October 2018, a total of 42 patients with known malignant gastrointestinal or pancreaticobiliary tumours were consecutively screened for LM (Figure 1). The local university ethics committee approved the study (application number 83/16). All participants gave their written informed consent to participate in the study. The study was conducted according to the principles of the Declaration of Helsinki (as revised in 2013) (27).

Of the 42 patients screened, no appropriate liver lesion could be detected in 10 patients by B-mode ultrasound. These patients were not further evaluated with CEUS in the study. The patients were excluded from the study. In 11 other patients, the liver lesions have been classified as benign on B-mode ultrasound. Another three patients 
Table 1 Patient characteristics, sonographic and histological results, and macroscopic semi-quantitative vascularisation results

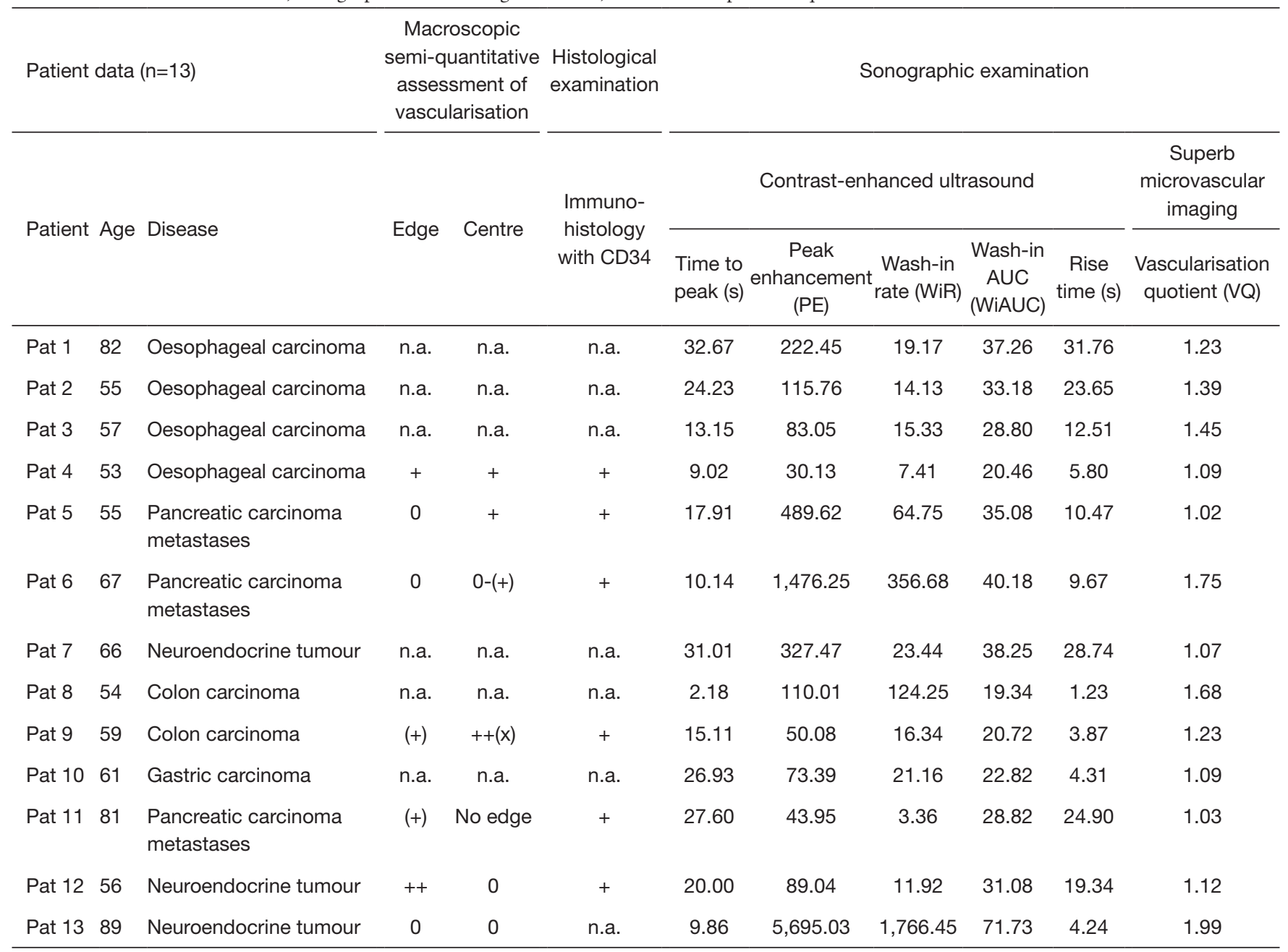

+, low vascularisation; ++, high vascularisation; n.a., no macroscopic or histological examination; 0 , no vascularisation. Shaded rows are patients with histological examinations.

were excluded, due to technical constraints that prevented lesion biopsy. In the further clinical course, the diagnosis of malignant liver lesions was confirmed in these patients. However, the clinical conditions of the patients also became so severe that participation in the study was no longer justifiable for ethical reasons at a later stage. Of the remaining 18 patients, no metastasis could be detected histologically after sonographic puncture in five additional patients (Figure 1).

A total of only 13 patients [ 6 women $/ 7$ men, mean age 64.2 years; (range, 53-89 years)] met the inclusion criteria: known tumour of the gastrointestinal tract or pancreaticobiliary system with hepatic metastasis. The mean body mass index was $25.2 \pm 5.4 \mathrm{~kg} / \mathrm{m}^{2}$. Of the 13 patients, only 6 could be punctured. Of the 13 patients included in the analysis, four patients had oesophageal carcinoma, three had pancreatic carcinoma, another three had neuroendocrine carcinoma, two had colon carcinoma, and one patient had gastric carcinoma (Table 1).

Gastrointestinal tract was defined as the esophagus from the superior esophageal sphincter, stomach, intestinum tenue, and intestinum crassum including anus to the external anal sphincter. The pancreaticobiliary system included the pancreas, gallbladder, and all bile ducts from their hepatic origin to their junction with the descending duodenal pars. The exclusion criteria were: pregnant and lactating women, children and adolescents under the age of 18 years, patients with recent history of acute coronary 
syndrome, clinically unstable ischemic heart disease, rightto-left cardiac shunts, severe pulmonary hypertension, uncontrolled systemic hypertension, or ARDS, as well as known intolerance to SonoVue ${ }^{\circledR}$.

For the final evaluation, LM had to be histologically confirmed ( $\mathrm{n}=6$ patients), or LM had to be clearly shown in the clinical course with CEUS, CT, or magnetic resonance tomography (MRI), without evidence of a second tumor of another origin ( $\mathrm{n}=7$ patients), histopathological confirmation, by means of puncture, was not performed in these 7 patients for ethical reasons (Figure 1).

\section{B-mode ultrasound}

The Aplio 500 ultrasound machine and the 6Cs1 transducer (1-6 MHz) from Toshiba (Minato, Tokyo, Japan) were used for the ultrasound examinations. All ultrasound examinations were performed after an overnight fasting period of at least $12 \mathrm{~h}$ by a single experienced investigator $(>5,000$ examinations per year). Images obtained during ultrasound examinations were digitally saved using the image backup program Viewpoint (GE Healthcare, Chalfont St. Giles, Buckinghamshire, England, UK), making them available for subsequent analysis and processing. B-scan ultrasonography of the liver was first performed in all patients. The focus of this examination was to describe the number, size, shape, pattern, and metastatic skin spacing of the metastases later examined quantitatively by CEUS and SMI. Localization was indicated with respect to liver segments according to Couinaud (28).

\section{Superb microvascular imaging monochrome (mSMI)}

In this study, monochrome mSMI was used exclusively to assess liver metastasis vascularisation because of its highest sensitivity (29-31). As also described by other authors, vascularisation can be better visualized in the socalled grayscale mode. In the mSMI mode, the parameters Doppler frequency, pulse repetition frequency, frame rate, and colour gain were set to the following mean settings during the study: $3.8 \mathrm{MHz}$ [Doppler frequency], $8.6 \mathrm{kHz}$ [pulse repetition frequency], frame rate $>30 \mathrm{fps}$, and colour gain 35. According to current literature, mSMI values were classified as follows: no vascularisation $(-)$, moderate vascularisation $(+)$, and strong vascularisation $(++)(32,33)$.

\section{Contrast enhanced ultrasound (CEUS)}

Contrast-enhanced examinations were performed using the "bolus injection method" according to the recommendations of the EFSUMB (34). The contrast agent used was SonoVue ${ }^{\circledR}$ (Bracco, Milan, Italy) $1.6 \mathrm{~mL}$ applied via intravenous access of a cubital vein with a $20 \mathrm{G}$ indwelling vein cannula over a period of two seconds and followed by $10 \mathrm{~mL}$ of an isotonic sodium chloride solution. Preferably, the left arm was used to improve sonic conditions after elevation of the right arm for examination of the liver. To minimize motion artifacts, patients were required to hold their breath as much as possible during the first twenty seconds of the recording and then to breathe as shallowly as possible; this had been practiced with them prior to the examination. The background for this is that when there are low breath-hold-related motion artifacts in the arterial phase, the quantitative $\mathrm{VueBox}^{\circledR}$ evaluation is improved (35).

The mechanical index (MI) was set between 0.05-0.09 and performed in dual-window mode. Total gain was set at 45 , dynamic range between $60-65 \mathrm{~dB}$, and frame rate between 10-15 fps. One focus zone was set below the liver metastasis. The video recording of the CEUS examination started with the bolus injection and was continuously captured as a DICOM file over $120 \mathrm{~s}$ while focusing on the liver metastasis.

\section{VueBox ${ }^{\circledR}$}

The contrast response of the liver metastasis and adjacent liver tissue were quantified using the CEUS evaluation program VueBox ${ }^{\otimes}$ (version 5.1, subprogram Liver DVP for focal liver lesions) (35). After loading the DICOM file, the video was optimally calibrated in terms of sampling rate, ultrasound system, dual display mode, and meant time gain compensation (Figure 2). The footage was then cropped for editing using the Clip Editor module, and unsuitable frames were deleted using Flash image capture. In B-scan mode ROI 1 was then placed over the liver metastasis and ROI 2, at the same level in the adjacent liver parenchyma. Specific care was taken to ensure that no major vessels or ligamentous structures were located in the ROI 2. In the case of greatly varying FLL sizes, we chose the largest possible reference ROI in tumour-free liver tissue as the measurement of liver metastasis size ROI 1. ROI 2 was then placed as centrally as possible in the metastasis (Figure 2). After confirmation or correction of contrast arrival, perfusion data processing was performed in bolus model mode with obtaining the following perfusion parameters: peak enhancement (PE), Wash-in AUC (WiAUC), Wash- 

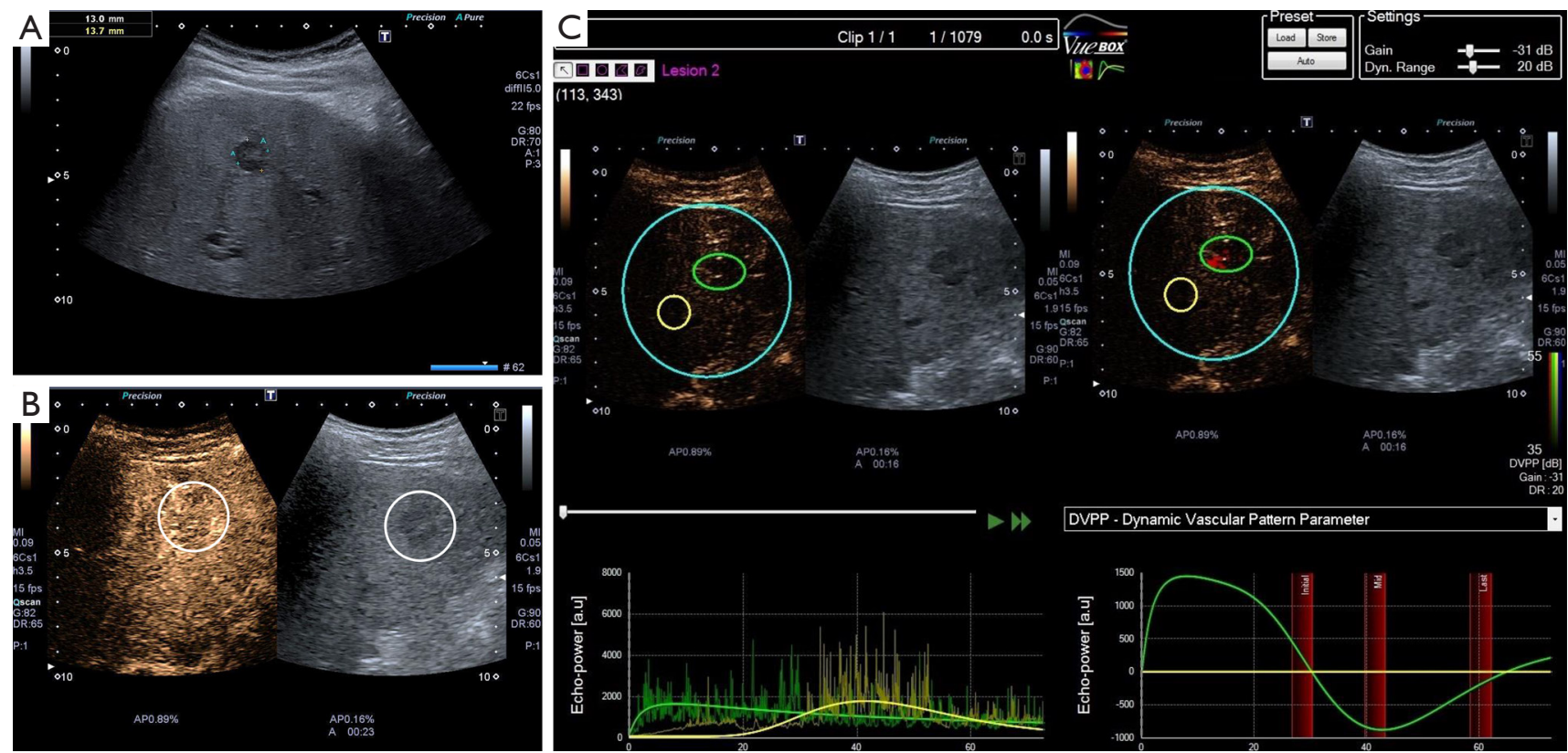

Figure 2 B-Mode ultrasound and CEUS imaging in a 67-year-old patient with confirmed pancreatic carcinoma metastases. (A) B-Mode ultrasound of the liver metastases (marked circular region). (B) Contrast-enhanced ultrasound, showing enhancement of the liver metastases (white circles); images were acquired at 23 s post-injection, arterial phase. (C) Corresponding results of the quantitative VueBox ${ }^{\circledR}$ analysis of the CEUS. The green curve corresponds to the examined metastasis and the yellow curve to the corresponding Reference ROI placed at the same level. The TIC shows the average intensity within a ROI displayed as a function of time which describes the wash-in and wash-out of the contrast agent. CEUS, contrast-enhanced ultrasound; TIC, time-intensity curve; ROI, region of interest.

in Rate (WiR), and Wash-in Perfusion Index (WiPI). A margin of error of $\pm 15 \%$ is reported for the vascularisation parameters of $\operatorname{VueBox}^{\circledR}(35)$. Furthermore, the Arrival Time (AT) reported by the investigator during CEUS examination was compared to that of VueBox ${ }^{\circledR}$. A difference of $1 \mathrm{~s}$ between the two different AT indications was considered acceptable.

\section{Imagef}

Image J software (version 1.51, open source, Bethesda, Maryland, USA) was used to perform grey-scale analyses of mSMI images of each FLL and the surrounding liver parenchyma (36). The mSMI images were saved as Joint Photographic (Experts) Group (JPG) files with vertical and horizontal resolutions of 72 dots per inch (dpi), a pixel count of $960 \times 720$, and a bit depth of 24 . Prior to the greyscale analysis, the ROI frame was placed over the FLL and positioned to include most of the FLL (Figure 3). The grey-scale analysis results were expressed both visually, as a histogram, and numerically, as the arithmetic mean, standard deviation, mode, minimum, maximum, and pixel count. Subsequently, a second ROI frame was positioned in the liver parenchyma, taking the pixel count of the first ROI into account, and taking care to avoid including prominent vessels. To account for any depth-dependent grey-scale changes in the image, the second area of evaluation was set at the same height as the first ROI, and the results were expressed as stated above. Five measurements were taken within the ROI at each of the ROI 1 and ROI 2 positions. Finally, the vascularisation quotient (VQ) was calculated from the mean values of the 5 measurements of ROI 1 and ROI 2 of grey-scale analysis (pixel intensity) as follows: VQ = metastasis intensity/liver parenchyma intensity (37) (Figure 4).

\section{MVD}

Among the 13 LMs, only 6 could be biopsied. The puncture specimens were examined histologically on a multi-head microscope to visualise vascularisation in a semi quantitative manner. All staining sections were performed with a microscope ${ }^{\circledR}$ (Olympus: BX41 Standard; 

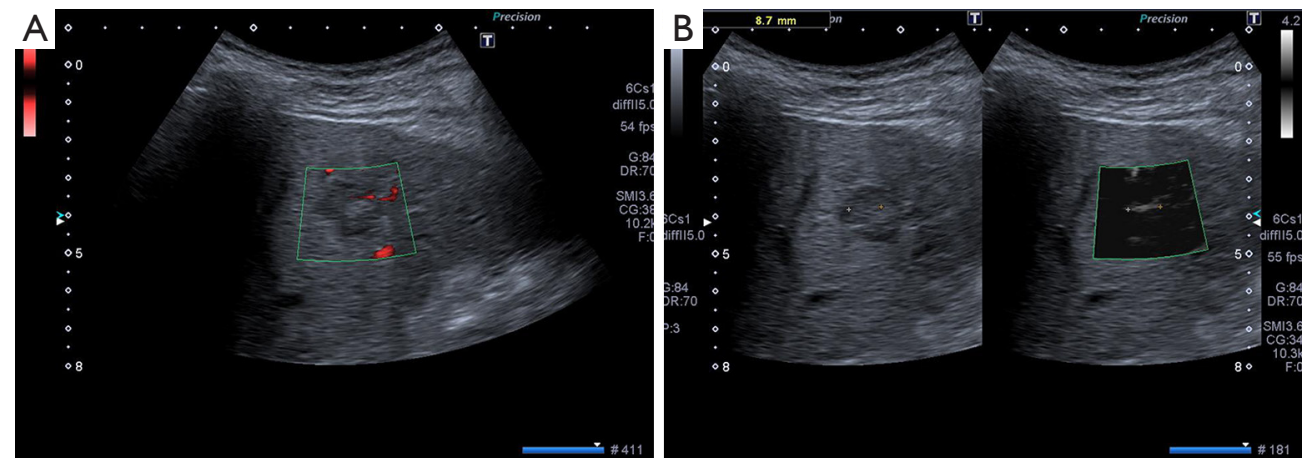

Figure $3 \mathrm{SMI}$ in a 67-year-old patient with confirmed pancreatic carcinoma metastases. The green box shows the region of interest. (A) Colour SMI of the liver metastasis. (B) Monochrome SMI of the liver metastasis. SMI, superb microvascular imaging.

Olympus Deutschland GmbH, Hamburg). The assessment of the vascularisation of the microvsacular density was performed in three degrees of expression. The degree of vascularisation was rated "0" (no vascular involvement), “+” (low vascularisation), or “++" (high vascularisation). Tumour neoangiogenesis was quantified based on the MVD. Specimens preserved in paraffin blocks were cut into 4 - $\mu \mathrm{m}$-thick sections and stained with haematoxylin-eosin or the monoclonal anti-CD34 antibody (1:50 dilution, M7165, clone QBEnd-10; Dako, A/S, DK) (19) (Table 1, Figures 5,6).

\section{Statistical analysis}

All statistical analyses were performed with SAS Version 9.4 software (SAS Institute, Cary, North Carolina, USA) and Microsoft Excel (Microsoft Corporation, Redmond, Washington, USA). A correlation between the results of the mSMI quantification with ImageJ and CEUS quantification with VueBox ${ }^{\circledR}$ was determined using a Pearson correlation. Potential confounding variables such as age, gender and body mass index were taken into account in the partial correlation. The level of significance was set at $\alpha=0.05$. P values $<0.05$ were considered significant.

\section{Results}

\section{Ultrasound findings}

The smallest FLL detected by ultrasound measured $9 \mathrm{~mm}$, and the largest measured $111 \mathrm{~mm}$, in diameter. Further examinations showed that 7 (53.8\%) FLLs had clearly defined margins, and $6(46.2 \%)$ had an inhomogeneous internal echo. Five FLLs (38.5\%) were hyperechoic, two $(15.4 \%)$ were isoechogenic, and six $(46.2 \%)$ were hypoechoic. In 5 (38.5\%) patients, a so-called halo sign could be visualized in the FLL. In one (7.7\%) patient, the LM was centrally anechoic. In another $(7.7 \%)$ patient, the metastasis exhibited calcifications. In $6(46.2 \%)$ patients, ultrasound showed only one LM, although in one $(7.7 \%)$ patient there were at least $10 \mathrm{LMs}$. The mean skin-tumour distance was $4.53 \pm 1.56 \mathrm{~cm}$ (range, $1.40-6.40 \mathrm{~cm}$ ).

\section{$m S M I$}

We analysed the grey-scale data from the mSMI Doppler ultrasound with ImageJ. The grey-scale analyses of FLLs suspected of malignancy were compared to grey-scale analyses of adjacent liver tissue not suspected of malignancy. The mean VQ and standard deviation were $1.318 \pm 0.314$, with a minimum of 1.02 and a maximum of 1.99 (Table 1).

\section{CEUS}

The CEUS evaluated the perfusion of FLLs suspected of malignancy. The CEUS quantification in VueBox ${ }^{\circledR}$ yielded values for the peak enhancement (PE) $22.41 \pm 6.50 \mathrm{~dB}$ (range, 14.79-37.55), wash-in area under the curve (WiAUC) $30.59 \pm 7.85 \mathrm{~dB}$ (range 19.34-41.73), wash-in rate (WiR) $15.16 \pm 7.38 \mathrm{~dB}$ (range, 5.26-32.47) and wash-in perfusion index (WiPI) $20.73 \pm 6.51 \mathrm{~dB}$ (range, 12.82-35.45) [Table 2 (38)].

\section{mSMI and CEUS}

The analysis revealed a statistically significant correlation of the parameters quantified in Image J (mSMI) compared to VueBox ${ }^{\circledR}$ (CEUS). The VQ showed a strong correlation with the quantified CEUS parameters $\mathrm{PE}$ in a.u. $(\mathrm{r}=0.72104$, 


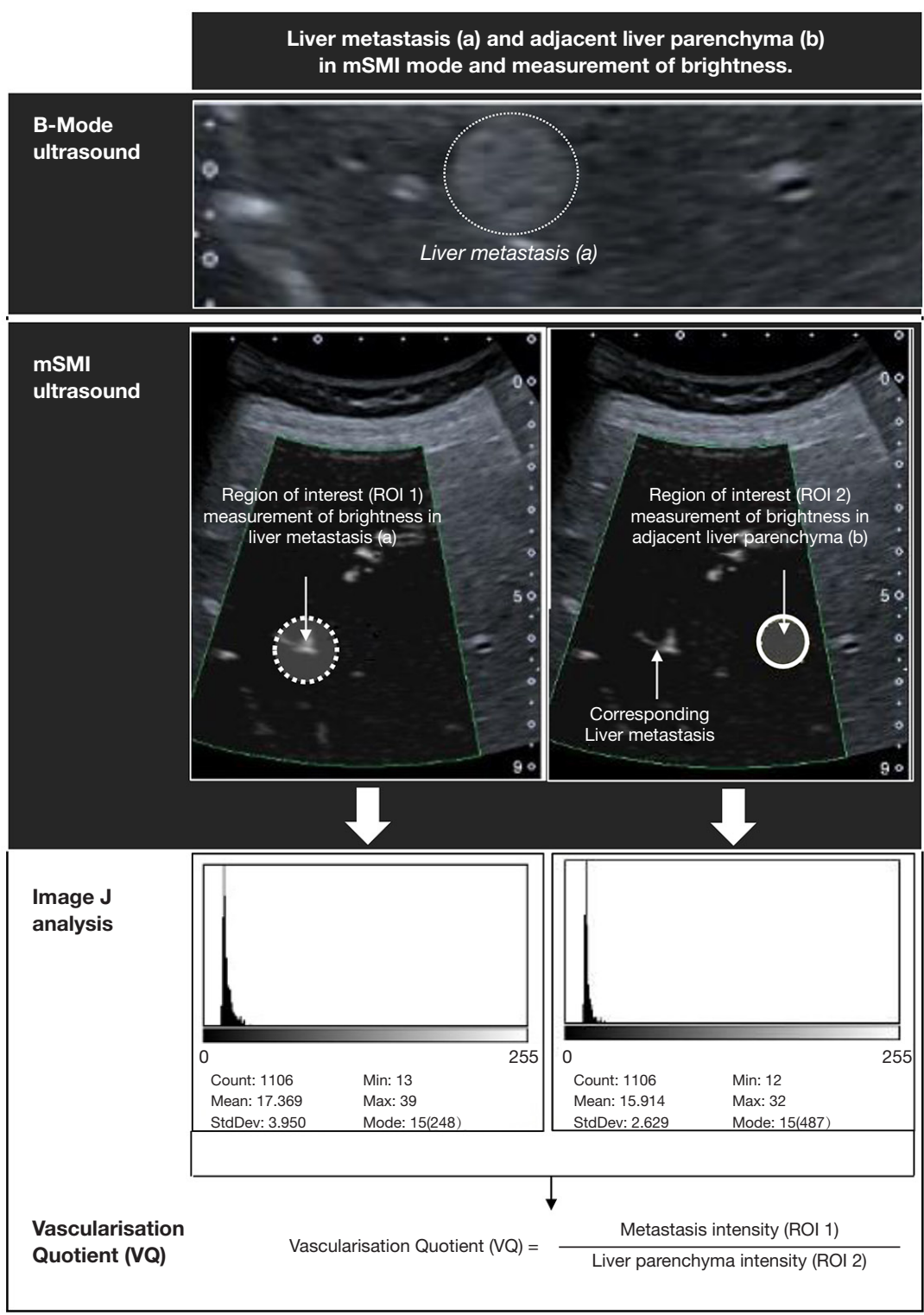

Figure 4 The figure shows the ultrasound images of a metastasis and of the adjacent liver parenchyma in B-mode and in mSMI. The ratio of liver metastasis to adjacent liver parenchyma forms the vascularisation quotient (VQ). The visualisation and analysis of the grey-scale within the ROIs in mSMI Mode was performed using ImageJ. (A) Analysis of a liver metastasis. (B) Analysis of the adjacent liver parenchyma. White circles: The ROI. ImageJ analysis: the white box presents the results of the ImageJ analysis. The scale shows the range of grey-scale values, defined in 256 increments from 0 (black) to 255 (white). In the analysis, a grey scale value is allocated to each pixel. Count: number of pixels evaluated; Min: the blackest (and thus darkest) grey scale value; Mean: the mean grey scale value; Max: the whitest (and thus brightest) grey scale value; StdDev: standard deviation of the grey scale values around the mean value; Mode: mode (in parentheses is the number of pixels whose grey-scale value corresponds to each mode). mSMI, monochromatic superb microvascular imaging; VQ, vascularisation quotient; ROI, region of interest. 


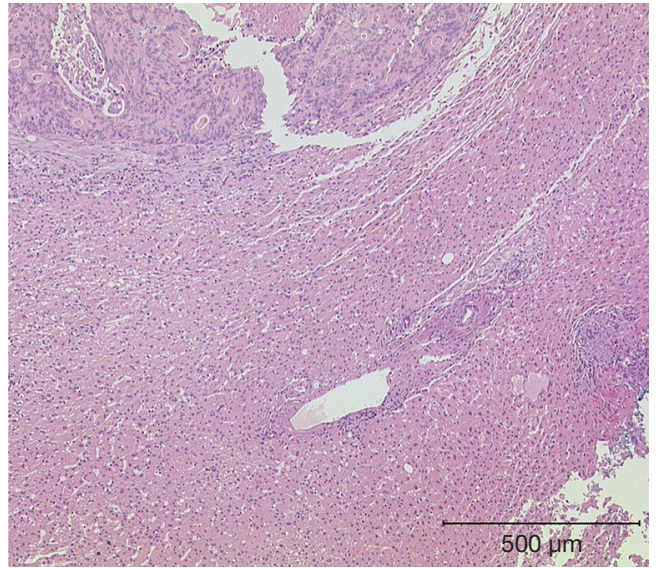

Figure 5 Histopathology of a liver metastasis. Conventional HE staining of a liver section.

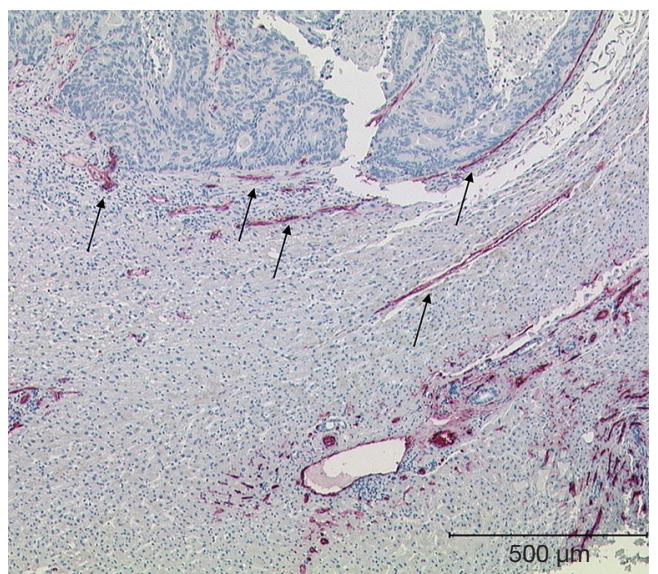

Figure 6 Histopathology of a liver metastasis. Immunohistological staining of a liver section, where the metastasis was detected with the CD34 antibody. The staining shows an increase in the density of neovessels around the metastasis (arrows).

$\mathrm{P}=0.0054), \mathrm{PE}$ in $\mathrm{dB}(\mathrm{r}=0.65918, \mathrm{P}=0.00141)$, WiAUC in a.u. $(\mathrm{r}=0.63604, \mathrm{P}=0.00194), \mathrm{WiPI}$ in a.u. $(\mathrm{r}=0.73337$, $\mathrm{P}=0.0043)$, $\mathrm{WiPI}$ in $\mathrm{dB}(\mathrm{r}=0.65642, \mathrm{P}=0.0194), \mathrm{WiR}$ in a.u. $(\mathrm{r}=0.7304, \mathrm{P}=0.0036)$ and $\mathrm{WiR}$ in $\mathrm{dB}(\mathrm{r}=0.82897, \mathrm{P}=0.0005)$ [Table 3 (38)].

\section{MVD}

We performed microscopic and immunohistological analyses of the MVD in tissue sections of the biopsy specimens. We found that neuroendocrine tumours had higher peripheral vascularisation than pancreatic metastases
Table 2 VueBox ${ }^{\circledR}$ results of the contrast-enhanced ultrasound (CEUS) examinations (38)

\begin{tabular}{lcc}
\hline Parameter & Mean \pm SD & Min-Max \\
\hline PE in a.u. & $677.40 \pm 1,557.10$ & $30.13-5,695.03$ \\
PE in dB & $22.41 \pm 6.50$ & $14.79-37.55$ \\
WiAUC in a.u. & $3,532.40 \pm 4,641.46$ & $85.91-14,888.37$ \\
WiAUC in dB & $30.59 \pm 7.85$ & $19.34-41.73$ \\
WiPI in a.u. & $438.63 \pm 964.51$ & $19.16-3,507.34$ \\
WiPI in dB & $20.73 \pm 6.51$ & $12.82-35.45$ \\
WiR in a.u. & $188.03 \pm 483.90$ & $3.36-1,766.45$ \\
WiR in dB & $15.16 \pm 7.38$ & $5.26-32.47$ \\
VQ (no unit) & $1.318 \pm 0.314$ & $1.02-1.99$ \\
\hline
\end{tabular}

PE, peak enhancement; WiAUC, wash-in area under the curve; WiPI, wash-in perfusion index; WiR, wash-in rate; $\mathrm{dB}$, decibels; a.u., arbitrary units.

(Table 1).

\section{Discussion}

To the best of our knowledge, this study was the first to evaluate mSMI and Image J for the characterisation of LMs compared to quantitative CEUS. Our SMI results showed a stronger signal in the LM compared to the normal liver parenchyma. We also found strong statistically significant correlations between the VQ quantified in $\mathrm{mSMI}$ with ImageJ and the quantified CEUS parameters from VueBox ${ }^{\circledR}$.

In a recent study on the value of quantitative CEUS for assessing malignant and benign FLLs, the greatest difference between benign and malignant lesions was observed in the peak enhancement. However, due to the time-consuming, complicated analysis with VueBox ${ }^{\circledR}$, those authors expected that CEUS would present considerable difficulties in clinical routine (10). Recent studies have demonstrated the superiority of SMI over colour and power Doppler sonography $(4,21)$.

Despite the superiority of SMI, especially monochrome SMI, several questions remain about SMI and need to be addressed in the future. Commercially, Canon's superb microvascular Doppler technology has been available since 2014 (29). The color-coded Doppler sonography and power Doppler technologies remove clutter by suppressing low velocity components. This suppression results in a loss of image data, and subsequent lost visibility of flow in smaller 
Table 3 Correlations between the VQ and quantified contrast-enhanced ultrasound parameters considering BMI, age, and sex (38)

\begin{tabular}{lcccc}
\hline Correlation of VQ with & Correlation coefficient $(r)$ & P value & Fisher's z $^{\text {. }}$ & 95\% confidence interval \\
\hline PE in a.u. & 0.72104 & 0.0054 & 0.90391 & $0.276706-0.909341$ \\
PE in dB & 0.65918 & 0.0141 & 0.79206 & $0.170582-0.887888$ \\
WiAUC in a.u. & 0.63604 & 0.0194 & 0.75135 & $0.130799-0.878952$ \\
WiAUC in dB & 0.30633 & 0.3087 & 0.31575 & $-0.295010-0.733169$ \\
WiPI in a.u. & 0.73337 & 0.0043 & 0.93018 & $0.300786-0.913781$ \\
WiPI in dB & 0.65642 & 0.0194 & 0.78718 & $0.165842-0.886851$ \\
WiR in a.u. & 0.74304 & 0.0036 & 0.95090 & $0.319517-0.917137$ \\
WiR in dB & 0.82897 & 0.0005 & 1.18942 & $0.515085-0.947752$ \\
\hline
\end{tabular}

VQ, vascularisation quotient; PE, peak enhancement; WiAUC, wash-in area under the curve; WiPI, wash-in perfusion index; WiR, wash-in rate; $\mathrm{dB}$, decibels; a.u., arbitrary unit. ${ }^{1}, 95 \%$ confidence intervals based on Fisher z-transformation.

vessels (39). By contrast, SMI analyzes the characteristics of movement artifacts arising from nearby structures and successfully extracts the clinically relevant information. Then, SMI uses another adaptive wall filter to suppress clutter noise and minimize the flash artifact. Consequently, SMI is expected to detect very low-velocity blood flow within the lesion $(19,29,39)$. Despite the superiority of SMI standards for normal perfusion patterns do not yet exist. The definition of thresholds for SMI, remain to be defined in future studies (19).

In a comparative study between SMI and CEUS in breast tumours, SMI showed a higher sensitivity than CEUS in the determination of blood flow signals. Conversely, in evaluating the malignant vessel pattern criteria, root-hairlike and crab claw-like, no significant differences were found between the two methods (40). However, the comparative studies carried out to date on SMI and other methods, such as colour- or power-Doppler sonography and CEUS, have been based exclusively on semi quantitative assessments, and the findings were subject to the experience and abilities of the investigators $(4,21,22,28,40)$. To what extent SMI, in particular monochrome SMI, is suitable for the differentiation of benign focal liver lesions cannot yet be conclusively answered. However, the characterization of FNHs could be promising for the application of SMI. Further studies will be needed to show whether quantification is necessary in this case (22). In the daily routine clarification of unclear liver lesions, SMI can quickly detect hyper vascularisation, especially in monochrome SMI mode. A further advantage remains the complete noninvasiveness of the method. Contrast-enhanced sonography will remain indispensable in the characterization of unclear hepatic lesions, where contrast agent infiltration and outflow are of crucial importance. In the control of masses, especially if it is only to assess vascularisation, e.g., for the condition after microwave ablation, we see a further possible application of SMI. Another use of SMI could be in the follow-up of stent grafts to exclude endoleaks after aortic aneurysm stenting. In a recent study SMI could be concretely used in the follow-up phase to increase colour Doppler ultrasound accuracy especially in patients who cannot be studied with CEUS or CT (41). The complementary role of antibody-determining MVD was demonstrated in two recent studies for differentiating tumour endpoints $(24,42)$.

The present pilot study had several limitations. First, we included a small number of patients. Consequently, no correlation was detected between the MVD of LMs and the measured SMI ratios. Second, the grey-scale analysis depended on the characteristics of the parenchyma, because the presence of larger vessels in the ROI would increase the measured values. A further limitation is the large range of FLLs that have been included into the study. Third, visualisation of the vascularisation with SMI also depended on the characteristics of the parenchyma (42). A fundamental problem of the study remains to what extent the increased brightness measured in the metastases actually corresponds to increased vascularisation. However, the currently available comparative studies between SMI and other Doppler techniques seem to confirm that the significantly better visualization of vascular structures by SMI corresponds to the increased measurable brightness in our study. To the best of our knowledge there are also no systematic studies on brightness and flow rate for SMI-monochrome applications. 
The clinical benefit for daily practice or other indications of a possible application of mSMI quantification can currently still be assessed (33,43-45).

Another limitation, which could lead to a possible misinterpretation of the results, is the fact that metastases of neuroendocrine tumours are mostly hyper vascularized in contrast enhanced ultrasound (CEUS) (46).

\section{Conclusions}

In conclusion, we showed a significant correlation between the quantified CEUS results and mSMI results, quantified with ImageJ. We also showed that quantified $\mathrm{mSMI}$ allowed an evaluation of the FLL perfusion status. Our results suggest that SMI could be useful for monitoring the efficacy of targeted anti-angiogenic therapies for the treatment of LM or other issues where the sole assessment of vascularisation yes/no or only the quantification of vascularisation is sufficient to answer the question (47). For the question of tumor characterisation, where inflow and outflow behavior are crucial for diagnosis, we currently see no application for SMI.

\section{Acknowledgments}

Funding: None.

\section{Footnote}

Conflicts of Interest: All authors have completed the ICMJE uniform disclosure form (available at https://dx.doi. org/10.21037/qims-21-383). The authors have no conflicts of interest to declare.

Ethical Statement: The authors are accountable for all aspects of the work in ensuring that questions related to the accuracy or integrity of any part of the work are appropriately investigated and resolved. All participants gave their written informed consent to participate in the study. The study was conducted according to the principles of the Declaration of Helsinki (as revised in 2013). The local Ethics Committee approved the study (application number 83/16).

Open Access Statement: This is an Open Access article distributed in accordance with the Creative Commons Attribution-NonCommercial-NoDerivs 4.0 International License (CC BY-NC-ND 4.0), which permits the non- commercial replication and distribution of the article with the strict proviso that no changes or edits are made and the original work is properly cited (including links to both the formal publication through the relevant DOI and the license). See: https://creativecommons.org/licenses/by-nc-nd/4.0/.

\section{References}

1. Kaltenbach TE, Engler P, Kratzer W, Oeztuerk S, Seufferlein T, Haenle MM, Graeter T. Prevalence of benign focal liver lesions: ultrasound investigation of 45,319 hospital patients. Abdom Radiol (NY) 2016;41:25-32.

2. Cosgrove DO. Malignant liver disease. In: Meire HB, Cosgrove DO, Dewbury KC, Farrant P, editors. Clinical ultrasound: a comprehensive text. Abdominal and general ultrasound, 2nd edition. London: Curchill Livingstone, 2001:211-31.

3. Westwood M, Joore M, Grutters J, Redekop K, Armstrong N, Lee K, Gloy V, Raatz H, Misso K, Severens J, Kleijnen J. Contrast-enhanced ultrasound using SonoVue ${ }^{\circledR}$ (sulphur hexafluoride microbubbles) compared with contrastenhanced computed tomography and contrast-enhanced magnetic resonance imaging for the characterisation of focal liver lesions and detection of liver metastases: a systematic review and cost-effectiveness analysis. Health Technol Assess 2013;17:1-243.

4. Bae JS, Lee JM, Jeon SK, Jang S. Comparison of MicroFlow Imaging with color and power Doppler imaging for detecting and characterizing blood flow signals in hepatocellular carcinoma. Ultrasonography 2020;39:85-93.

5. Lee SY, Rhee CM, Leung AM, Braverman LE, Brent GA, Pearce EN. A review: Radiographic iodinated contrast media-induced thyroid dysfunction. J Clin Endocrinol Metab 2015;100:376-83.

6. Isaka Y, Hayashi H, Aonuma K, Horio M, Terada Y, Doi $\mathrm{K}$, et al. Guideline on the use of iodinated contrast media in patients with kidney disease 2018. Clin Exp Nephrol 2020;24:1-44.

7. Tsushima Y, Kanal E, Thomsen HS. Nephrogenic systemic fibrosis: risk factors suggested from Japanese published cases. Br J Radiol 2010;83:590-5.

8. Kanda T, Fukusato T, Matsuda M, Toyoda K, Oba H, Kotoku J, Haruyama T, Kitajima K, Furui S. Gadoliniumbased Contrast Agent Accumulates in the Brain Even in Subjects without Severe Renal Dysfunction: Evaluation of Autopsy Brain Specimens with Inductively Coupled Plasma Mass Spectroscopy. Radiology 2015;276:228-32. 
9. Beckett KR, Moriarity AK, Langer JM. Safe Use of Contrast Media: What the Radiologist Needs to Know. Radiographics 2015;35:1738-50.

10. Wildner D, Schellhaas B, Strack D, Goertz RS, Pfeifer L, Fiessler C, Neurath MF, Strobel D. Differentiation of malignant liver tumors by software-based perfusion quantification with dynamic contrast-enhanced ultrasound (DCEUS). Clin Hemorheol Microcirc 2019;71:39-51.

11. Goertz RS, Bernatik T, Strobel D, Hahn EG, Haendl T. Software-based quantification of contrast-enhanced ultrasound in focal liver lesions--a feasibility study. Eur J Radiol 2010;75:e22-6.

12. Schellhaas B, Bernatik T, Bohle W, Borowitzka F, Chang J, Dietrich CF, et al. Contrast-Enhanced Ultrasound Algorithms (CEUS-LIRADS/ESCULAP) for the Noninvasive Diagnosis of Hepatocellular Carcinoma - A Prospective Multicenter DEGUM Study. Ultraschall Med 2021;42:e20.

13. Schwarze V, Marschner C, Völckers W, Grosu S, Negrão de Figueiredo G, Rübenthaler J, Clevert DA. Diagnostic value of contrast-enhanced ultrasound versus computed tomography for hepatocellular carcinoma: a retrospective, single-center evaluation of 234 patients. J Int Med Res 2020;48:300060520930151.

14. Schwarze V, Marschner C, Negrão de Figueiredo G, Rübenthaler J, Clevert DA. Single-Center Study: Evaluating the Diagnostic Performance and Safety of Contrast-Enhanced Ultrasound (CEUS) in Pregnant Women to Assess Hepatic Lesions. Ultraschall Med 2020;41:29-35.

15. Yang F, Zhao J, Liu C, Mao Y, Mu J, Wei X, Jia J, Zhang S, Xin X, Tan J. Superb microvascular imaging technique in depicting vascularity in focal liver lesions: more hypervascular supply patterns were depicted in hepatocellular carcinoma. Cancer Imaging 2019;19:92.

16. Joo I, Kim SH, Lee DH, Han JK. Dynamic ContrastEnhanced Ultrasound of Gastric Cancer: Correlation with Perfusion CT and Histopathology. Korean J Radiol 2019;20:781-90.

17. Averkiou MA, Bruce MF, Powers JE, Sheeran PS, Burns PN. Imaging Methods for Ultrasound Contrast Agents. Ultrasound Med Biol 2020;46:498-517.

18. Dujardin PA, Léger J, Lecomte T, Patat F, Chassagnon G, Bleuzen A. Perfusion Quantification of Liver Metastases of Colorectal Cancer Treated with Anti-angiogenicBased Therapy: Assessment of Intra- and Inter-observer Reproducibility of Parameters in Three Regions of Interest Outlining Lesions. Ultrasound Med Biol 2020;46:286-96.
19. Jiang ZZ, Huang YH, Shen HL, Liu X'T. Clinical Applications of Superb Microvascular Imaging in the Liver, Breast, Thyroid, Skeletal Muscle, and Carotid Plaques. J Ultrasound Med 2019;38:2811-20.

20. Zhan J, Diao XH, Jin JM, Chen L, Chen Y. Superb Microvascular Imaging-A new vascular detecting ultrasonographic technique for avascular breast masses: A preliminary study. Eur J Radiol 2016;85:915-21.

21. Dubinsky TJ, Revels J, Wang S, Toia G, Sonneborn R, Hippe DS, Erpelding T. Comparison of Superb Microvascular Imaging With Color Flow and Power Doppler Imaging of Small Hepatocellular Carcinomas. J Ultrasound Med 2018;37:2915-24.

22. He MN, Lv K, Jiang YX, Jiang TA. Application of superb microvascular imaging in focal liver lesions. World J Gastroenterol 2017;23:7765-75.

23. Rueden CT, Schindelin J, Hiner MC, DeZonia BE, Walter AE, Arena ET, Eliceiri KW. ImageJ2: ImageJ for the next generation of scientific image data. BMC Bioinformatics 2017;18:529.

24. Bösmüller H, Pfefferle V, Bittar Z, Scheble V, Horger M, Sipos B, Fend F. Microvessel density and angiogenesis in primary hepatic malignancies: Differential expression of CD31 and VEGFR-2 in hepatocellular carcinoma and intrahepatic cholangiocarcinoma. Pathol Res Pract 2018;214:1136-41.

25. Paschoal JP, Bernardo V, Canedo NH, Ribeiro OD, Caroli-Bottino A, Pannain VL. Microvascular density of regenerative nodule to small hepatocellular carcinoma by automated analysis using CD105 and CD34 immunoexpression. BMC Cancer 2014;14:72.

26. Segatelli V, de Oliveira EC, Boin IF, Ataide EC, Escanhoela CA. Evaluation and comparison of microvessel density using the markers CD34 and CD105 in regenerative nodules, dysplastic nodules and hepatocellular carcinoma. Hepatol Int 2014;8:260-5.

27. General Assembly of the World Medical Association. World Medical Association Declaration of Helsinki: ethical principles for medical research involving human subjects. J Am Coll Dent 2014;81:14-8.

28. Wolf H, Gross F, Merz A, Schuler A. Liver segment anatomy in ultrasound--examinations to define the frontier between segment II/III and literature review. Z Gastroenterol 2013;51:271-7.

29. Hata J. Medical review: Seeing the unseen: new techniques in vascular imaging. Toshiba Medical Systems. 2014: Available online: https://pdfs.semanticscholar.org/cd4b/ b692fc285cc65791de0034c0229fc3411daa.pdf. Accessed 
October 10, 2019.

30. Machado P, Segal S, Lyshchik A, Forsberg F. A Novel Microvascular Flow Technique: Initial Results in Thyroids. Ultrasound Q 2016;32:67-74.

31. Ohno Y, Fujimoto T, Shibata Y. A New Era in Diagnostic Ultrasound, Superb Microvascular Imaging: Preliminary Results in Pediatric Hepato-Gastrointestinal Disorders. Eur J Pediatr Surg 2017;27:20-5.

32. Adler DD, Carson PL, Rubin JM, Quinn-Reid D. Doppler ultrasound color flow imaging in the study of breast cancer: preliminary findings. Ultrasound Med Biol 1990;16:553-9.

33. Kloth C, Eissler A, Schmidberger J, Gräter T, Scheuerle A, Kratzer W, Pedro MT. Quantitative Analysis of Superb Microvascular Imaging versus Color-Coded Doppler Sonography for Preoperative Evaluation of Vascularization of Schwannomas. J Neurol Surg A Cent Eur Neurosurg 2020;81:213-9.

34. Dietrich CF, Averkiou MA, Correas JM, Lassau N, Leen E, Piscaglia F. An EFSUMB introduction into Dynamic Contrast-Enhanced Ultrasound (DCE-US) for quantification of tumour perfusion. Ultraschall Med 2012;33:344-51.

35. Tranquart F, Mercier L, Frinking P, Gaud E, Arditi M. Perfusion quantification in contrast-enhanced ultrasound (CEUS)--ready for research projects and routine clinical use. Ultraschall Med 2012;33 Suppl 1:S31-8.

36. Fortin M, Battié MC. Quantitative paraspinal muscle measurements: inter-software reliability and agreement using OsiriX and ImageJ. Phys Ther 2012;92:853-64.

37. Zhang XY, Zhang L, Li N, Zhu QL, Li JC, Sun Q, Wang HY, Jiang YX. Vascular index measured by smart 3-D superb microvascular imaging can help to differentiate malignant and benign breast lesion. Cancer Manag Res 2019;11:5481-7.

38. Dobler F. Evaluation of tumor heterogeneity in patients with hepatic metastatic tumors using different ultrasound modalities (B-scan, color Doppler, elastography, and contrast-enhanced ultrasound). Open Access Repository of the University of Ulm and Ulm University of Technology. Dissertation. 2019. Available online: http://dx.doi. org/10.18725/OPARU-11357

39. Park AY, Seo BK. Up-to-date Doppler techniques for breast tumor vascularity: superb microvascular imaging and contrast-enhanced ultrasound. Ultrasonography 2018;37:98-106.

40. Xiao XY, Chen X, Guan XF, Wu H, Qin W, Luo BM. Superb microvascular imaging in diagnosis of breast lesions: a comparative study with contrast-enhanced ultrasonographic microvascular imaging. Br J Radiol 2016;89:20160546.

41. Cantisani V, David E, Ferrari D, Fanelli F, Di Marzo L, Catalano C, Benedetto F, Spinelli D, Katsargyris A, Blandino A, Ascenti G, D'Ambrosio F. Color Doppler Ultrasound with Superb Microvascular Imaging Compared to Contrast-enhanced Ultrasound and Computed Tomography Angiography to Identify and Classify Endoleaks in Patients Undergoing EVAR. Ann Vasc Surg 2017;40:136-45.

42. Kuroda H, Abe T, Kakisaka K, Fujiwara Y, Yoshida Y, Miyasaka A, Ishida K, Ishida H, Sugai T, Takikawa Y. Visualizing the hepatic vascular architecture using superb microvascular imaging in patients with hepatitis $C$ virus: $A$ novel technique. World J Gastroenterol 2016;22:6057-64.

43. Keçeli M, Keskin Z, Keskin S. Comparison of Superb Microvascular Imaging With Other Doppler Methods in Assessment of Testicular Vascularity in Cryptorchidism. Ultrasound Q 2020;36:363-70.

44. Ayaz E, Aslan A, İnan İ, Yikılmaz A. Evaluation of Ovarian Vascularity in Children by Using the "Superb Microvascular Imaging" Ultrasound Technique in Comparison With Conventional Doppler Ultrasound Techniques. J Ultrasound Med 2019;38:2751-60.

45. Durmaz MS, Sivri M. Comparison of superb microvascular imaging (SMI) and conventional Doppler imaging techniques for evaluating testicular blood flow. J Med Ultrason (2001) 2018;45:443-52.

46. Massironi S, Conte D, Sciola V, Pirola L, Paggi S, Fraquelli M, Ciafardini C, Spampatti MP, Peracchi M. Contrast-enhanced ultrasonography in evaluating hepatic metastases from neuroendocrine tumours. Dig Liver Dis 2010;42:635-41.

47. Pregler B, Beyer LP, Wiesinger I, Nießen C, Jung EM, Stroszczynski C, Wiggermann P. Microwave ablation of large HCC lesions: Added value of CEUS examinations for ablation success control. Clin Hemorheol Microcirc 2016;64:483-90.

Cite this article as: Kratzer W, Güthle M, Dobler F, Seufferlein T, Graeter T, Schmidberger J, Barth TFE, Klaus J. Comparison of superb microvascular imaging (SMI) quantified with Image J to quantified contrast-enhanced ultrasound (qCEUS) in liver metastases-a pilot study. Quant Imaging Med Surg 2022;12(3):1762-1774. doi: 10.21037/qims-21-383 